\title{
The damping of slow MHD waves in solar coronal magnetic fields
}

\section{The effect of mode coupling}

\author{
I. De Moortel, A. W. Hood, C. L. Gerrard, and S. J. Brooks
}

\begin{abstract}
School of Mathematics and Statistics, University of St Andrews, North Haugh, St Andrews, Fife KY16 9SS, Scotland
e-mail: ineke@mcs.st-and.ac.uk
\end{abstract}

Received 5 March 2004 / Accepted 22 June 2004

\begin{abstract}
The properties of slow MHD waves in a two dimensional model are investigated, in a low-beta plasma. Including a horizontal density variation causes "phase mixing" and coupling between slow and fast MHD waves. The effects of different density profiles, different driving frequencies, different values for the viscosity coefficient and plasma beta $(<1)$ are studied. Using numerical simulations, it was found that the behaviour of the perturbed velocity was strongly dependent on the values of the parameters. From analytical approximations, a strong interaction with the fundamental, normal modes of the system was found to play an important role. The coupling to the fast wave proved to be an inefficient way to extract energy from the driven slow wave and is unlikely to be responsible for the rapid damping of propagating slow MHD waves, observed by TRACE. The "phase mixing" of the slow waves due to the (horizontal) density inhomogeneity does cause a significant amount of damping, but is again unlikely to be sufficiently strong to explain the rapid observed damping.
\end{abstract}

Key words. Sun: oscillations - Sun: corona - Sun: activity

\section{Introduction}

Since the launch of SOHO and TRACE, many examples of small amplitude oscillations have been detected in a variety of solar structures, including the propagating slow MHD waves on which we will concentrate in this paper. Ofman et al. (1997) first detected periodic density variations in coronal plumes, using UVCS/SOHO. Similar perturbations in plumes were also found in EIT/SOHO observations by DeForest \& Gurman (1998). Ofman et al. (1999, 2000) found that these quasi-periodic disturbances could be modelled as slow magneto-acoustic waves, propagating along coronal plumes. Similar periodic, propagating disturbances have been observed in coronal loops, both by EIT/SOHO (Berghmans \& Clette 1999) and TRACE (De Moortel et al. 2000). Robbrecht et al. (2001) and King et al. (2003) presented a comparison of the properties of the density variations in the EIT/SOHO $195 \AA$ and TRACE $171 \AA$ passbands, whereas Nakariakov et al. (2000), followed by Tsiklauri \& Nakariakov (2001), constructed a model in terms of slow magneto-acoustic waves. These latter authors also show that wide-spectrum slow magneto-acoustic waves could provide a sufficient rate of heat deposition to heat the coronal loops in which the perturbations are observed. A similar study was carried out earlier by Erdélyi (1996). De Moortel et al. (2002a,b) presented an extensive overview and discussion of the properties of these observed longitudinal intensity oscillations in coronal loops.
This paper is the third in a series of papers looking at the damping of slow magneto-acoustic waves in the solar corona. The previous two papers (De Moortel \& Hood 2003, 2004) investigated the properties of uncoupled slow MHD waves, from the point of view of boundary driven oscillations, in a 1D model. These authors found that, for the observed coronal conditions, thermal conduction appeared to be the dominant damping mechanism, when compared to the effect of compressive viscosity and optically thin radiation. The model was extended to include gravitational stratification and a diverging magnetic field and it was demonstrated that a general area divergence can cause a significant, additional, decrease of the amplitudes of the perturbations. A combination of thermal conduction and (general) area divergence yielded detection lengths that are in good agreement with observed values. The first two papers in this series more or less exhausted the possibilities of a 1D, isothermal, loop model and we now investigate a two dimensional model. This allows us to incorporate a horizontal density variation, which will lead to the excitation of different MHD modes and, hence, energy could leave the system as it is transferred to a different wave mode.

The coupling of different wave modes has been investigated by many authors, in a wide variety of settings and with varying levels of complexity. Among the first to consider the coupling of different wave modes in the solar atmosphere are Ferraro \& Plumpton (1958). The problem was re-addressed by Zhugzhda \& Dzhalilov (1984), in terms of Meijer G-functions, and later by Cally (2001), who pointed out an alternative 
solution in terms of (less daunting) hypergeometric ${ }_{2} F_{3}$ functions. However, all these authors consider mode coupling through a vertical density inhomogeneity, i.e. gravitational stratification.

Mode coupling will also occur through a horizontal density inhomogeneity but so far, most of the studies have concentrated on the coupling between fast and Alfvén waves, often in the context of phase mixing of Alfvén waves (Heyvaerts \& Priest 1983). For example, Nocera et al. (1984) considered nonlinear coupling of Alfvén waves and magneto-acoustic waves, propagating in the longitudinal direction, whereas Nakariakov et al. (1997) studied the nonlinear excitation of fast magnetoacoustic waves by phase mixed Alfvén waves, in an inhomogeneous, cold plasma. Subsequently, Nakariakov et al. (1998) investigated the nonlinear coupling of MHD waves when a inhomogeneous flow, directed along the magnetic field, is present. Numerical simulations, presented by Botha et al. (2000) showed that the nonlinear coupling of Alfvén waves to fast waves is actually quite inefficient. The efficiency was estimated to be proportional to the square of the Alfvén wave amplitude (Malara et al. 1996; Botha et al. 2000). Some features observed (but not explained) in the simulations by Botha et al. (2000) are also seen in this study, and will be discussed in detail later. Cally \& Maddison (1997) describe the evolution of oscillations when a horizontal density inhomogeneity is present, in terms of spatial Fourier modes. The authors consider the wave evolution in a rigid box and, mainly focus on the cascade of energy to progressively smaller spatial scales due to the process of phase mixing.

In the literature, the term "phase mixing" usually refers to "phase mixing of Alfvén waves". As Alfvén waves are unable to propagate across the magnetic field, there is no interaction between neighbouring field lines and hence, it is possible for disturbances to propagate along different field lines at different speeds. The propagating waves will quickly become out of phase and large (horizontal) gradients will build up. This allows the energy transfer to small lengthscales, where eventually dissipation can become important. However, slow magnetoacoustic waves exhibit largely the same anisotropic behaviour as Alfvén waves. When a density inhomogeneity is present, the slow waves on different field lines will propagate at different speeds, leading to a very similar process of "phase mixing". It is this "phase mixing of slow waves" that is referred to in this paper. However, unlike the Alfvén waves, the slow waves are slightly dispersive, and hence, are not entirely confined to propagation strictly along the magnetic field lines. This different, physical property of the slow waves, will lead to a different form of phase mixing.

A very detailed and comprehensive set of numerical simulations of wave propagation through the (stratified) solar atmosphere can be found in Rosenthal et al. (2002) and Bogdan et al. (2003). The authors mainly concentrate on the solar photosphere and chromosphere and demonstrate that the coupling between slow and fast modes is most effective where the sound and Alfvén speed are comparable in magnitude, i.e. where the plasma beta approaches unity.

The main aim of this paper is not an in-depth study of the coupling between the various MHD modes as they propagate through the various layers of the solar atmosphere. Instead, we will investigate the linear coupling of boundary driven slow waves to fast waves, through a transversal (horizontal) density inhomogeneity, in a low-beta (coronal) plasma. This will enable us to determine the contribution of mode coupling and phase mixing to the rapid damping of slow magneto-acoustic waves, observed in large coronal loops. To some extend, one can consider our numerical simulations to be a subset of the complex work presented by Rosenthal et al. (2002) and Bogdan et al. (2003). However, our more simplified approach allows us to isolate the effect of a horizontal density inhomogeneity and to obtain analytical approximations to provide a deeper understanding of the basic physical processes that are occuring. The basic equations and model are presented in Sect. 2, whereas a description of the results of the numerical simulations is given in Sect. 3. Subsequently, several analytical approximations are studied in Sect. 4, followed by a comparison of observed slow MHD waves and conclusions (Sect. 5).

\section{Description of the model}

\subsection{Basic equations}

For our simple model, the equilibrium is given by a constant, vertical background magnetic field, $\boldsymbol{B}_{0}=\left(0,0, B_{0}\right)$, an inhomogeneous density, $\rho=\rho_{0}(x)$, and a constant pressure, $p=p_{0}$. For both the analytical calculations and numerical simulations presented in this paper, we use the standard, linearised MHD equations,

$$
\begin{aligned}
& \rho_{0} \frac{\partial \boldsymbol{v}_{1}}{\partial t}=-\nabla p+\boldsymbol{j}_{1} \times \boldsymbol{B}_{0}+\rho_{0} v \nabla^{2} \boldsymbol{v}_{1}, \\
& \frac{\partial \rho_{1}}{\partial t}=-\nabla \cdot\left(\rho_{0} \boldsymbol{v}_{1}\right), \\
& \frac{\partial p_{1}}{\partial t}=-\gamma p_{0} \nabla \cdot \boldsymbol{v}_{1}, \\
& \frac{\partial \mathbf{b}_{1}}{\partial t}=\nabla \times\left(\boldsymbol{v}_{1} \times \boldsymbol{B}_{0}\right),
\end{aligned}
$$

where $\rho$ is the mass density, $p$ the gas pressure, $v$ the velocity, $j$ the current density and $b$ the magnetic field. Subscript " 1 " denotes perturbed quantities, whereas " 0 " refers to equilibrium quantities. The viscosity $v$ is assumed to be constant.

Before proceeding, Eqs. (1) to (4) are made dimensionless using the equilibrium values for pressure and density. Furthermore, length and time are non-dimensionalised in terms of a distance $L$ and time $t_{0}$ and the velocity is expressed as $v=v_{0} \bar{v}$, where $v_{0}=L / t_{0}$. The resulting system of equations contains a dimensionless viscosity parameter,

$\bar{v}=\frac{v}{\rho_{0} v_{0} L} \frac{1}{\bar{\rho}_{0}}$.

The dimensionless Alfvén speed and adiabatic sound speed are given by $\bar{v}_{\mathrm{A}}^{2}=1 / \bar{\rho}_{0}$ and $\bar{c}_{\mathrm{s}}^{2}=\gamma \beta / 2 \bar{\rho}_{0}=\bar{\beta} / \bar{\rho}_{0}$, respectively (where $\bar{\beta}=\gamma \beta / 2$ ).

In this work we study both the slow and fast MHD oscillations, in a horizontally inhomogeneous medium. Hence, we consider a density profile that has a gradient in the $x$-direction, $\rho_{0}(x)$, and assume a vertical, background magnetic field $B_{0}$, 

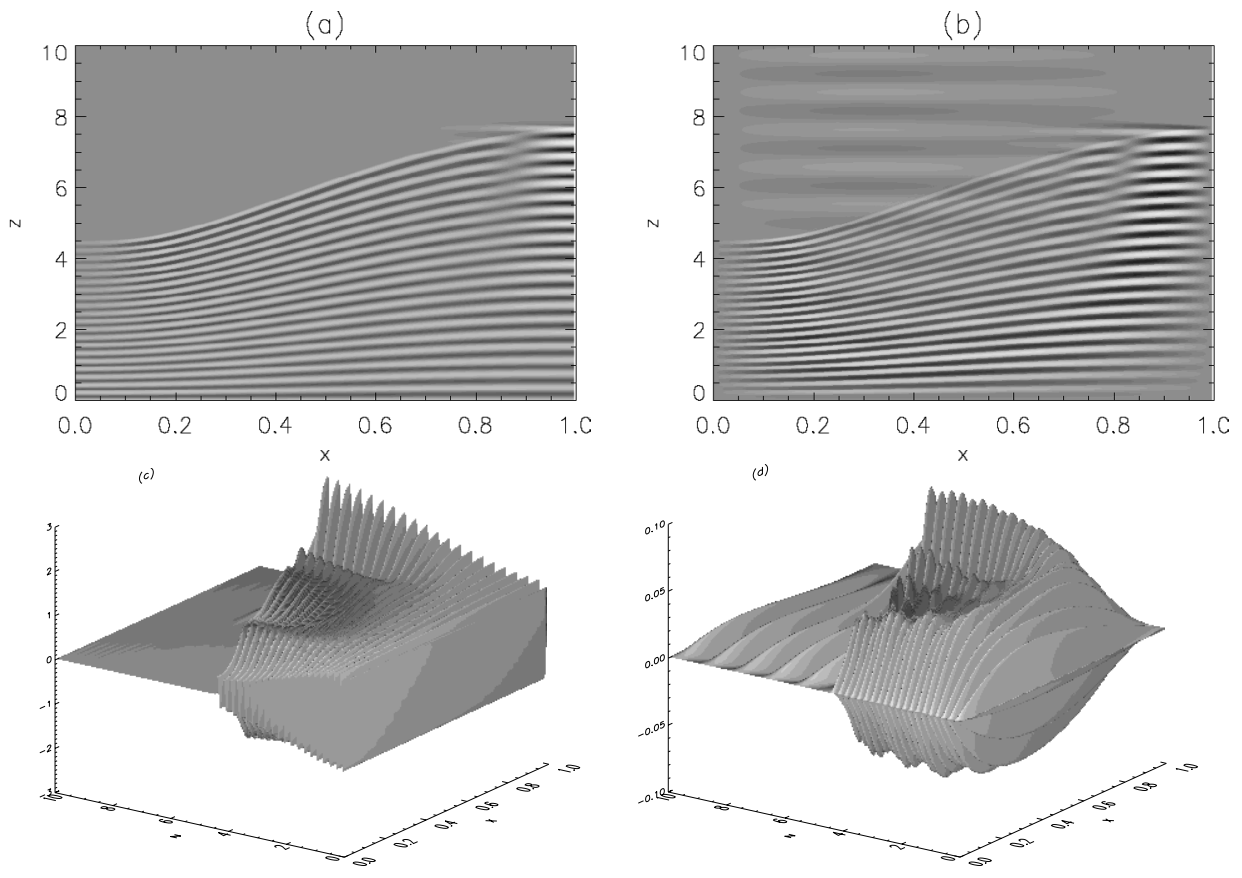

Fig. 1. a) A contour plot of the $z$ component of the perturbed velocity, $v_{z}$, at $t=20.2$, with $\varepsilon=0.5, \beta=0.1$ and $\omega=2 \pi$. b) Corresponding contour plot of the $x$ component of the perturbed velocity, $v_{x}$. c)-d) Similar to a) and b) but surface plots. Note the difference in the horizontal and vertical scales in these figures.

directed along the $z$-axis. Considering perturbations to the velocity and magnetic field in both the $x$ and $z$-directions and dropping bars from dimensionless quantities, Eqs. (1) to (4) can be combined to give

$$
\begin{aligned}
& \frac{\partial^{2} v_{z}}{\partial t^{2}}=c_{\mathrm{s}}^{2}\left(\frac{\partial^{2} v_{x}}{\partial z \partial x}+\frac{\partial^{2} v_{z}}{\partial z^{2}}\right)+v \nabla^{2} \frac{\partial v_{z}}{\partial t}, \\
& \frac{\partial^{2} v_{x}}{\partial t^{2}}=\left(c_{\mathrm{s}}^{2}+v_{\mathrm{A}}^{2}\right) \frac{\partial^{2} v_{x}}{\partial x^{2}}+c_{\mathrm{s}}^{2} \frac{\partial^{2} v_{z}}{\partial x \partial z}+v_{\mathrm{A}}^{2} \frac{\partial^{2} v_{x}}{\partial z^{2}}+\nu \nabla^{2} \frac{\partial v_{x}}{\partial t} .
\end{aligned}
$$

The inhomogeneous density profile is obtained by setting

$\rho_{0}=(1-\varepsilon \cos (\pi x))^{-1}$,

where the parameter $\varepsilon$ controls the gradient of the inhomogeneous density profile.

\subsection{Numerical code}

The system of equations described above is solved numerically, using a centred, sixth-order finite-difference scheme, with a third-order Runge-Kutta based time-step. We use a computational domain where the (horizontal) $x$-component lies between 0 and 1 , whereas the (vertical) $z$-component lies between 0 and 15. This implies that the width of the coronal structure will determine the basic lengthscale in our simulations. For all simulations, we used 200 gridpoints in the horizontal direction, and 1600 in the vertical direction, which was sufficient to resolve the increasingly small lengthscales, as the boundary-driven disturbances propagated (and phase mixed) with height.
Initially, at $t=0$, all variables are set equal to zero. The boundary conditions are taken as follows:

$$
\begin{aligned}
& \text { - At } z=0, \\
& \quad-v_{z}(x, 0, t)=\sin (\omega t), \\
& \quad-v_{x}(x, 0, t)=0 . \\
& \text { - At } x=0 \text { and } x=1, \\
& \quad-\frac{\partial v_{z}}{\partial x}(0, z, t)=\frac{\partial v_{z}}{\partial x}(1, z, t)=0, \\
& \quad-v_{x}(0, z, t)=v_{x}(1, z, t)=0 .
\end{aligned}
$$

\section{Results of numerical simulations}

In this section, we describe the results of the numerical simulations in several steps. Initially, we set the viscosity coefficient $v=0$, i.e. we consider an ideal plasma, and investigate the effect of varying the inhomogeneity parameter $\varepsilon$ (Sect. 3.1), the driving frequency (Sect. 3.2), and the plasma beta (Sect. 3.3). Subsequently, we will study the effect of viscosity by setting $v \neq 0$ (Sect. 3.4).

\subsection{Varying the density profile}

As pointed out before, the density, and hence the Alfvén speed varies in the horizontal direction. From Eq. (8), it is clear that the speed is lowest for $x=0$, i.e. at the left hand side of the numerical box, and highest at $x=1$, or the right hand side. This will result in a turning of the wavefront, as different parts of the wave travel at different speeds. The larger the value of $\varepsilon$, the stronger the density inhomogeneity and hence the turning of the wavefront. Figures $1 \mathrm{a}$ and $1 \mathrm{~b}$ show a contour plot of both the $z$ and $x$ components of the perturbed velocity at a time 

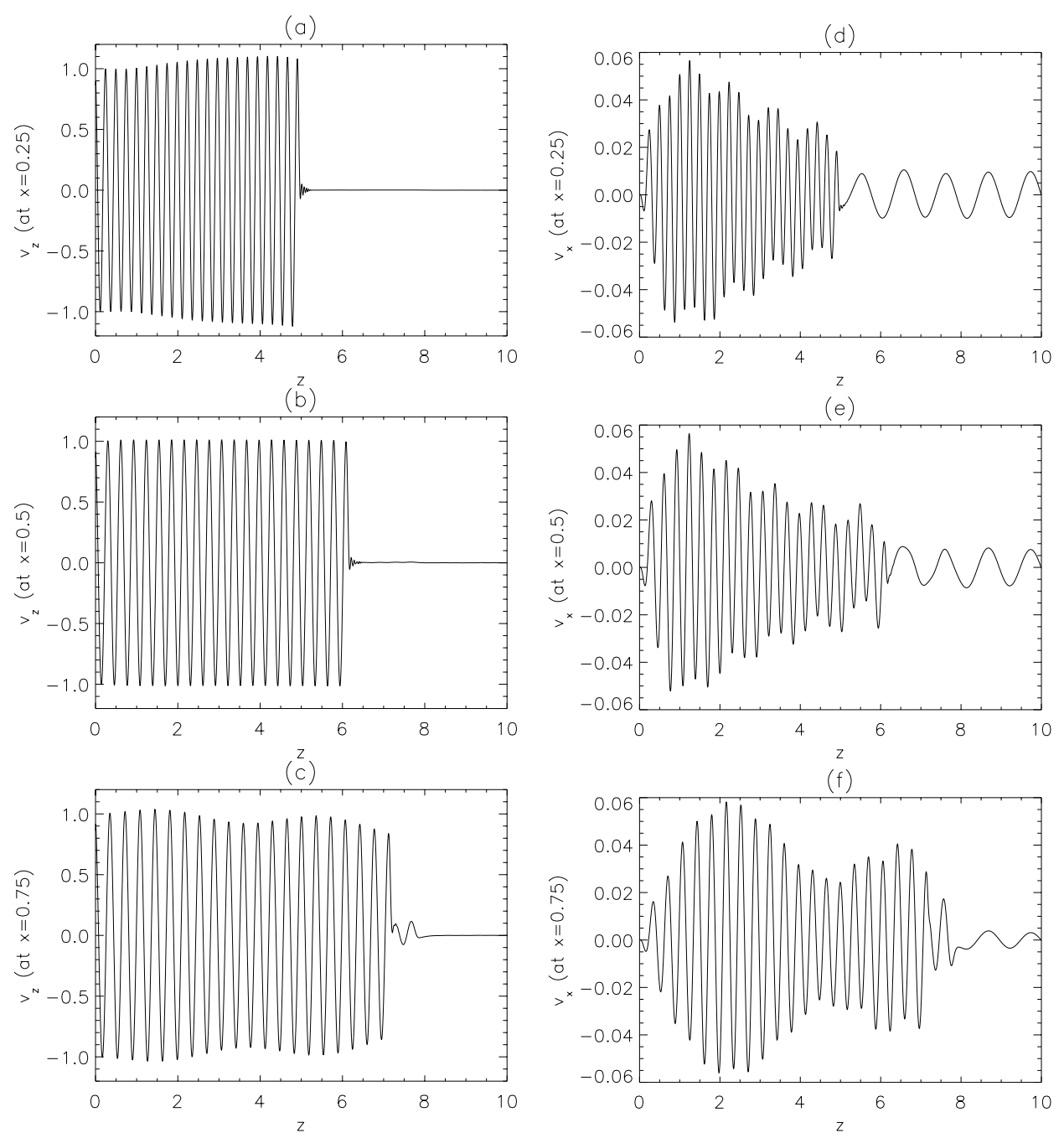

Fig. 2. a) A cross-section of the $z$ component of the perturbed velocity, $v_{z}$, at $x=0.25$ and $t=20.2$, with $\varepsilon=0.5, \beta=0.1$ and $\omega=2 \pi$. b)-c) Similar to a) but at $x=0.5$ and $x=0.75$, respectively. d)-f) Corresponding cross-sections of the $x$ component of the perturbed velocity, $v_{x}$.

$t=20.2$ and with $\varepsilon=0.5$ and $\beta=0.1$. For both components, the strong turning of the wavefront is obvious (especially if one keeps in mind that the $x$ and $z$ axis are scaled differently). From these graphs, we see that there are two additional features present. Firstly, there appears to be a disturbance that is confined to the right hand side of the domain and which is present in both the $z$ and $x$ components. The wavelength of this disturbance appears to be similar to that of the driven disturbance. Secondly, there is an additional, longer-wavelength, disturbance present in the $x$ component, which has its maximum in the left half of the domain. Contrary to the boundary-driven wave, the wavefront of this additional disturbance does not appear to turn, indicating that its propagation speed is largely independent of $x$. The surface plots of Figs. 1c and 1d emphasise the different wavelenghts that are present (especially in $v_{x}$ ) and the complicated pattern of the amplitudes of both $v_{z}$ and $v_{x}$. As the perturbed velocity propagates up in height, the wavefront does not only turn, but the amplitudes of the components build up a strong variation in both the $x$ and the $z$ direction.

Figure 2 shows cross-sections of both the $z$ and $x$ component of the perturbed velocity, at different values of $x$. Firstly, we observe that there is relatively little change in the amplitude of $v_{z}$, for all values of $x$. At $x=0.5$, there is no significant change in the amplitude of $v_{z}$. For very small $x$ (i.e. near the left-hand border of the computational domain), the amplitude of $v_{z}$ decreases with height. Further away from this boundary, but for $x$ smaller than 0.5 there is a small increase in amplitude. For $x$ larger than 0.5 , the amplitude appears to be modulated by the presence of a beat. Finally, for $x$ near the right-hand boundary, the amplitude of the perturbed velocity increases with height.

Reducing the value of $\varepsilon$ to 0.1 and 0.05 results in a behaviour of $v_{z}$ that is only slightly different. For these smaller values of $\varepsilon$, the amplitude of $v_{z}$ at $x=0.5$ no longer remains constant but decreases, and the decrease gets larger for smaller values of $\varepsilon$. At small $x$, there is now an initial decay before the amplitude increases again. The height at which certain features occur in the numerical results increases as $\varepsilon$ is decreased. For example, the modulation of the amplitude at larger $x$ still occurs, but the wavelength of the modulation increases with decreasing $\varepsilon$. 
(a)
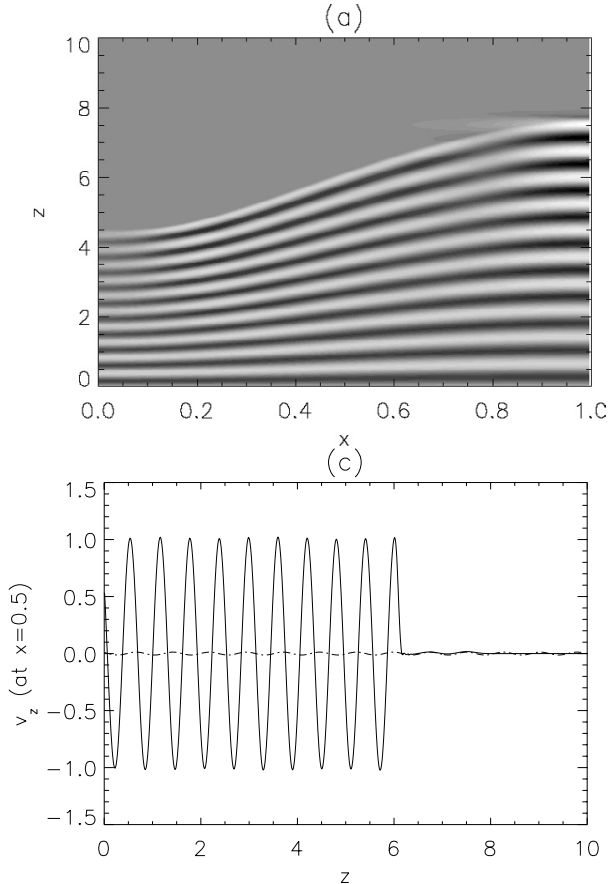

(b)

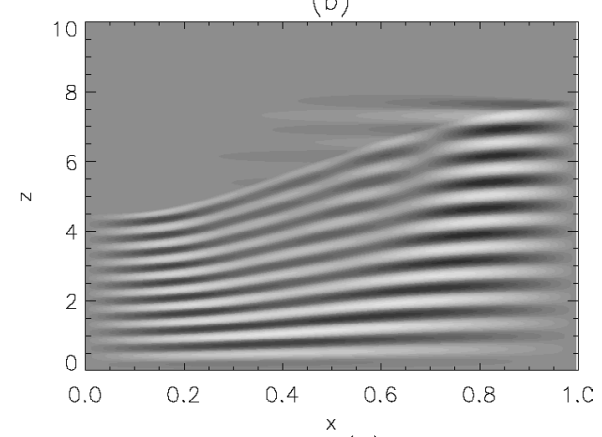

(d)

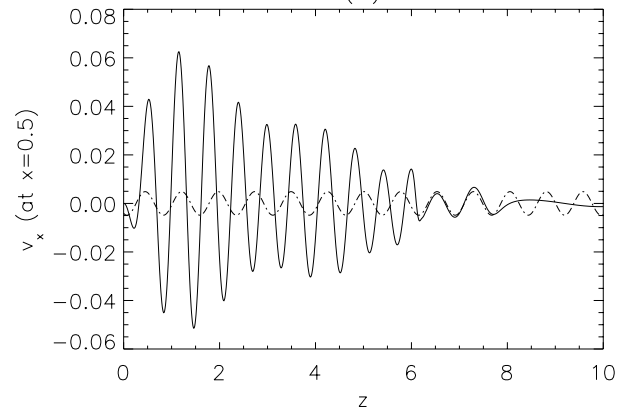

Fig. 3. a) A contour plot of the $z$ component of the perturbed velocity, $v_{z}$, at $t=38.2$, with $\varepsilon=0.5, \beta=0.1$ and $\omega=\pi$. b) Corresponding contour plot of the $x$ component of the perturbed velocity, $v_{x}$. c)-d) Cross-sections at $x=0.5$ of the $z$ component and $x$ component, respectively. The dot-dashed lines correspond to analytical approximations which will be discussed in Sect. 4.3.

Both the size and the behaviour of the amplitude of the $x$ component are radically different. The amplitude of $v_{x}$ is slightly smaller for smaller $\varepsilon$, but the difference is not significant, and certainly does not scale as $\varepsilon$. For all values of $x$, there is a relatively rapid, initial growth, followed by a slower decline in amplitude. The turn-around occurs at smaller heights for smaller values of $x$ and for larger values of $\varepsilon$. Figures $2 \mathrm{~d}-\mathrm{f}$ show that the amplitude of the longer-wavelength disturbance is slightly smaller for larger values of $x$. Additionally, the amplitude of this fast disturbance decreases with decreasing $\varepsilon$. Finally, we notice the presence of a strong beat in Fig. 2f. For smaller values of $\varepsilon$, this beat did not occur before the driven disturbance reached the boundary of our computational domain $\left(z_{\max }=15\right)$.

\subsection{Varying the driving frequency}

The numerical results shown in Fig. 3 are obtained for the same parameters as above $(\varepsilon=0.5, \beta=0.1)$ but the frequency of the boundary-driven disturbance has been decreased from $\omega=2 \pi$ to $\omega=\pi$. As the Alfvén speed profile remains unchanged, this lower frequency will result in smaller wavenumbers $\left(k=\omega / c_{\mathrm{s}}\right)$, and hence longer wavelengths $(\lambda=2 \pi / k)$, which is obvious when comparing Figs. 3 and 1. A more unexpected result of lowering the driving frequency, is that the longer-wavelength, fast disturbance does not appear to be present. The absence of this fast mode will be discussed in Sect. 4.2. Again comparing Figs. $3 \mathrm{~b}$ and $1 \mathrm{~b}$, we note that the slow disturbance is now situated further away from the right hand boundary, and more toward the centre of the domain (see discussion at the end of Sect. 4.3).
Figures $3 \mathrm{c}$ and $\mathrm{d}$ show cross-sections of both components of the perturbed velocity at $x=0.5$. From the corresponding cross-section for a larger driving frequency (Fig. 2b), we see that, apart from the longer wavelength, the behaviour of the $z$-components is very similar. The only slight difference is that the amplitude of the additional mode is a little larger when the driving frequency is smaller. A similar conclusion can be made for the $x$-component. From Figs. $3 \mathrm{~d}$ and 2e, we see that for this cross-section, the amplitudes of the $x$-components are of a similar size, where the amplitude of the large $\omega$ disturbance is marginally smaller. The turn-around point, where the amplitude of the $x$-component starts decreasing after the initial increase, is also located at roughly the same height. Finally, note that the additional mode, ahead of the driven disturbance (at $x=0.5$ ), is now the slow mode, rather than the fast mode, which explains why it has not yet reach the top boundary of the computational domain. This results might be seen more clearly from a comparison of Figs. $3 b$ and $1 b$.

\subsection{Varying the plasma beta}

Keeping the lower driving frequency, $\omega=\pi$, and the inhomogeneity coefficient, $\varepsilon=0.5$, we now decrease the plasma beta from $\beta=0.1$ to a value $\beta=0.01$. Figure 4 shows cross-sections at $x=0.5$ of both the $z$ and $x$ components of the perturbed velocity at a time $t=90.2$. The results described in Sect. 3.1 largely remain the same if we decrease the value of $\beta$ (to 0.05 and 0.01 ). Generally the turn-around in the behaviour of the $v_{x}$ amplitude (from growing to decaying) happens slightly lower down for smaller $\beta$. For $\varepsilon=0.5$, the same "beating" behaviour is seen in the amplitude of $v_{x}$ 
(a)

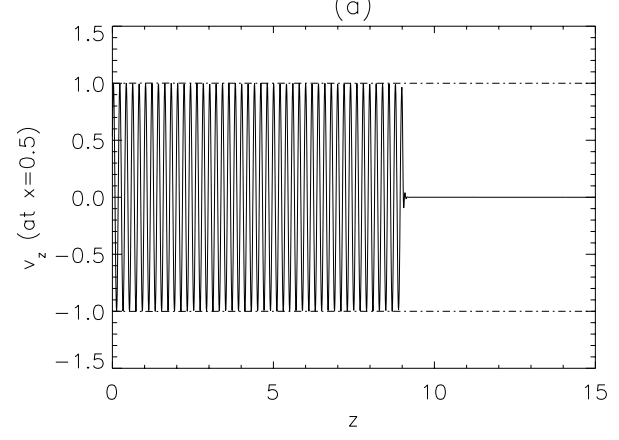

(b)

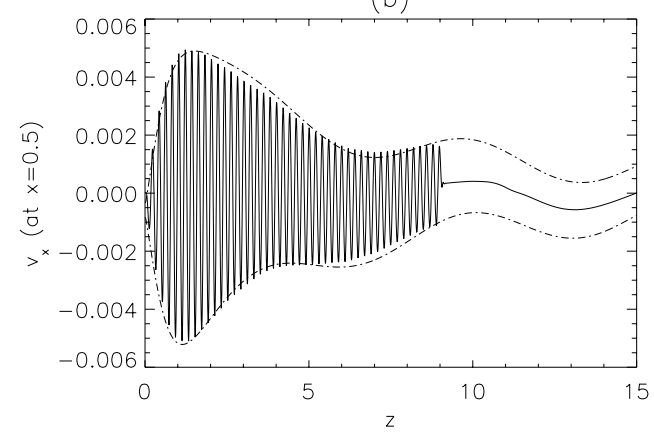

Fig. 4. a) A cross-section of the $z$ component of the perturbed velocity, $v_{z}$, at $x=0.5$ and $t=90.2$, with $\varepsilon=0.5, \beta=0.01$ and $\omega=\pi$. b) Corresponding cross-sections of the $x$ component of the perturbed velocity, $v_{x}$. The dot-dashed lines correspond to analytical approximations which will be discussed in Sect. 4.3.

(a)

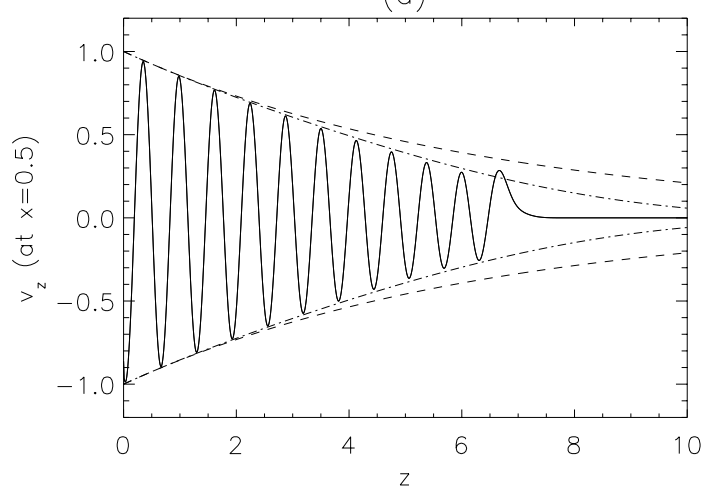

(b)

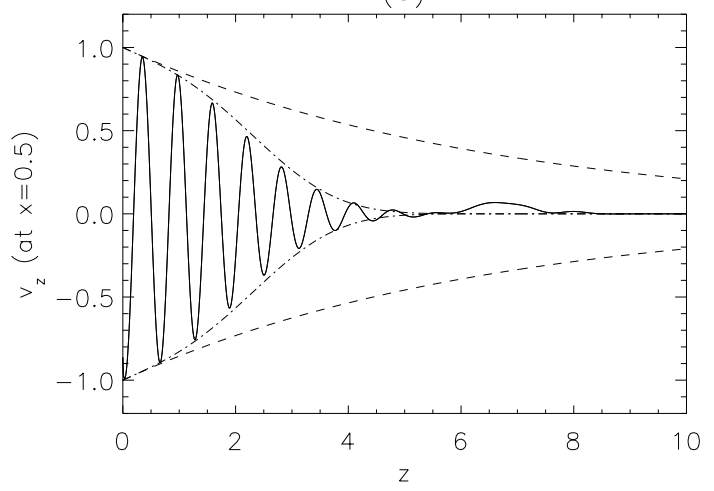

Fig. 5. a) A cross-section of the $z$ component of the perturbed velocity at $x=0.5$ and $t=21.6$, with $v=0.001, \varepsilon=0.1, \beta=0.1$ and $\omega=\pi$. The dot-dashed line corresponds to the predicted damping rate (Eq. (10)). The dashed line corresponds to the predicted damping rate for $\varepsilon=0$. (Eq. (11)) b) Similar to a) but with $\varepsilon=0.5$.

when $\beta=0.05$. For the smaller value of $\beta=0.01$, it was unclear whether the beating did no longer occur, or whether it did not occur inside our computational domain. The wavelength of the driven perturbations decreases for smaller $\beta$. Indeed, for a wavenumber $k=\omega / c_{\mathrm{s}}$, where $c_{\mathrm{s}}=\sqrt{\beta} v_{\mathrm{A}}$, the wavelength of the driven disturbance, given by $\lambda=2 \pi / k=2 \pi \sqrt{\beta} v_{\mathrm{A}} / \omega$, scales with the plasma beta. On the other hand, for this smaller value of $\beta$, the additional, faster propagating disturbance is again present, but with a considerably longer wavelength than in the $\beta=0.1, \omega=2 \pi$ case (compare Figs. $4 \mathrm{~b}$ and $2 \mathrm{e}$ ). The overall amplitude of $v_{x}$ varies with $\beta$, i.e. the amplitude of $v_{x}$ is roughly a magnitude smaller, when $\beta$ is reduced by a factor of ten. Finally, the variation in the $v_{z}$ amplitude also appears to scale with $\beta$, where for $\beta=0.01$ there was no longer a noticeable decrease of the amplitude, for any value of $x$ and for $\varepsilon=0.5$ or 0.1 . This suggests that the strength or efficiency of the mode coupling depends strongly on $\beta$. Intuitively, one can understand this in the following way. For smaller values of the plasma beta, pressure perturbations will become less important, and hence the generation of compressional disturbances less efficient.

\subsection{Varying the viscosity coefficient}

We now include viscosity in the model by setting the viscosity coefficient $v \neq 0$. Figure 5 shows the results of numerical simulations for $v=0.001$ and for different values of $\varepsilon$. The following, simple analytical argument gives an estimate of the damping rate due to the viscosity.

Using $v_{\mathrm{A}}^{2}=1 / \rho_{0}$ and $c_{\mathrm{s}}^{2}=\beta v_{\mathrm{A}}^{2}$ and setting the viscosity coefficient $v=v_{0} \beta^{2}$, the equation for the $z$ component of the perturbed velocity, Eq. (6), can be rewritten as

$-\rho_{0} \frac{\partial^{2} v_{z}}{\partial t^{2}}+\beta \frac{\partial^{2} v_{x}}{\partial z \partial x}+\beta \frac{\partial^{2} v_{z}}{\partial z^{2}}+\rho_{0} v \nabla^{2} \frac{\partial v_{z}}{\partial t}=0$.

To estimate the amplitude decay of the perturbed velocity, we assume

$v_{x}, v_{z} \sim \sin \left(\omega t-\frac{k(x) z}{\sqrt{\beta}}\right) \mathrm{e}^{\sqrt{\beta} \psi(x, z)}$

Equating the terms of order $\beta$ in Eq. (9) that contribute to the amplitude change results in

$\frac{\partial \psi}{\partial z}=-\frac{\omega v_{0} \rho_{0}}{2 k}\left(k^{2}+k^{\prime 2} z^{2}\right)$.

Hence, we expect an amplitude decay of the form

$v_{z} \sim \exp \left(-\frac{\omega v \rho_{0}}{2 k \beta \sqrt{\beta}}\left(k^{2} z+\frac{k^{\prime 2} z^{3}}{3}\right)\right)$. 
For a homogeneous density profile $(\varepsilon=0), k \sim \omega$ and $k^{\prime}=0$, and hence, the amplitude decrease takes the form

$v_{z} \sim \exp \left(-\frac{\omega^{2} v \rho_{0} z}{2 \beta \sqrt{\beta}}\right)$.

Firstly, we consider the dashed line in Fig. 5a, which corresponds to the damping rate (Eq. (11)) expected for a homogeneous density profile $(\varepsilon=0)$. In this case, there is no turning of the wavefront, and no mode coupling and any amplitude decay is purely due to viscosity. This estimated decay agreed well with a numerical simulation for which $\varepsilon=0$ but the numerical result was not included, in order not to make the figure needlessly complicated. If we compare this dashed line with the solid line, we see that the small density inhomogeneity $(\varepsilon=0.1)$ causes the amplitude of $v_{z}$ to decrease slightly quicker. The faster decay is due to the turning of the wavefront, which causes the build up of transversal gradients, and the generation of an $x$ component of the perturbed velocity. The dot-dashed line corresponds to the predicted damping rate (Eq. (10)), which agrees well with the numerical result. We also note here that this damping rate has the same form as the damping rate of phase mixed Alfvén waves (Heyvaerts \& Priest 1983), where at larger height, the behaviour will be mostly determined by the $\exp \left(-z^{3}\right)$ component.

Comparing the dot-dashed and dashed lines shows that, for this small value of $\varepsilon$, most of the amplitude decay is caused by the viscosity. Increasing $\varepsilon$ to 0.5 (Fig. 5b) leads to a much faster decay of the amplitude. Due to the steeper density inhomogeneity, large transversal gradients will be generated quicker, and the mode coupling will be stronger. Ahead of the decaying part of the wave is a propagating, transient pulse, as discussed by Hood et al. (2002). These authors demonstrate that such a pulse will decay at a slower, algebraic rate, rather than the exponential decay of an infinite wavetrain, as seen in Fig. 5. Finally we mention that the decrease in amplitude of the perturbed velocity is obviously stronger when the viscosity coefficient $v$ is increased.

\section{Analytical approximations}

Although the full Eqs. (6) and (7) cannot be solved analytically, we can make some progress by assuming that either the plasma beta, or the inhomogeneity coefficient $\varepsilon$ is small. We will use the approximate analytical solutions to explain some of the behaviour and features described in Sect. 3. Firstly, we will assume that the plasma beta is sufficiently small to allow us to expand both $v_{z}$ and $v_{x}$ in powers of $\sqrt{\beta}$.

\subsection{Small $\beta$ expansion}

The ideal, coupled equations for $v_{x}$ and $v_{z}$ are given by Eqs. (6) and (7), with $v=0$. Using $v_{\mathrm{A}}^{2}=1 / \rho_{0}$ and $c_{\mathrm{s}}^{2}=\beta v_{\mathrm{A}}^{2}$, these equations can be rewritten as

$$
\begin{aligned}
& -\rho_{0} \frac{\partial^{2} v_{z}}{\partial t^{2}}+\beta \frac{\partial^{2} v_{x}}{\partial z \partial x}+\beta \frac{\partial^{2} v_{z}}{\partial z^{2}}=0, \\
& -\rho_{0} \frac{\partial^{2} v_{x}}{\partial t^{2}}+(1+\beta) \frac{\partial^{2} v_{x}}{\partial x^{2}}+\beta \frac{\partial^{2} v_{z}}{\partial x \partial z}+\frac{\partial^{2} v_{x}}{\partial z^{2}}=0 .
\end{aligned}
$$

We now expand both $v_{z}$ and $v_{x}$ in powers of $\beta$ by setting

$$
\begin{aligned}
& v_{z}=\sin \left(\omega t-\frac{k(x) z}{\sqrt{\beta}}+\sqrt{\beta} \phi(x, z)\right), \\
& v_{x}=\beta A(x, z) \sin \left(\omega t-\frac{k(x) z}{\sqrt{\beta}}+\sqrt{\beta} \phi(x, z)\right),
\end{aligned}
$$

where $A(x, z)$ and $\phi(x, z)$ are to be determined. Using these expansions and the fact that $k^{2}=\omega^{2} / v_{A}^{2}=\omega^{2} \rho_{0}$, the leading order equation for $v_{x}$ is given by

$-k^{\prime 2} z^{2} A-k k^{\prime} z-k^{2} A=0$,

or

$A(x, z)=\frac{-k k^{\prime} z}{k^{2}+k^{\prime 2} z^{2}}$

From this expression for the coefficient $A$, we can see that the $x$-component of the perturbed velocity will remain zero (i.e. there is no mode coupling) when there is no density inhomogeneity present $\left(k^{\prime}=0\right)$.

Substituting expressions (14) and (15) into Eq. (12) and equating terms of order $\beta$ results in

$-k k^{\prime} z A+2 k \frac{\partial \phi}{\partial z}=0$,

or

$\frac{\partial \phi}{\partial z}=-\frac{1}{2} \frac{k k^{\prime 2} z^{2}}{k^{2}+k^{\prime 2} z^{2}}$

Integrating this expression with respect to $z$ gives the change in phase as

$\phi(x, z)=-\frac{1}{2} k z+\frac{k^{2} \arctan \left(k^{\prime} z / k\right)}{2 k^{\prime}}+C(x)$,

and finally, from $v_{z}(z=0)=\sin (\omega t)$, we find $C(x)=0$. For small $\beta$, the components of the perturbed velocity are now given by expressions (14) and (15), where $A(x, z)$ is defined in Eq. (16) and $\phi(x, z)$ in Eq. (17). Including viscosity in this derivation would result in the same expression for the amplitude change as given by Eq. (10).

Figure 6a shows the cross-section of $v_{x}$ at $x=0.5$ for small $\beta(=0.01)$ and for different values of $\varepsilon$. The dot-dashed and long-dashed lines outline the amplitude variations obtained from the corresponding analytical approximations. For this value of $\beta$ there is a good agreement between the numerical result and the analytical approximation. More specifically, the coefficient $A(x, z)$ seems to correctly predict the turn-around in the amplitude of $v_{x}$ for this small value of $\beta$. From setting $\partial A / \partial z=0$, the location of the maximum of the coefficient $A$ is given by $z_{\max }=k / k^{\prime}$, and from Fig. $6 \mathrm{~b}$ we see that this maximum will occur at smaller values of $z$ for larger $\varepsilon$. Comparing Figs. $6 \mathrm{a}$ and $\mathrm{b}$ confirms that the turn-around points for $\varepsilon=0.1$ and 0.5 are indeed located at 6.3 and 1.3 , respectively. As this is only a small-beta expansion, the variation of the turn-around point with $\beta$ can not be deduced. From expression (15), it is obvious that the amplitude of $v_{x}$ will indeed scale as $\beta$, as was found in the numerical simulations discussed in Sect. 3.3. We found an equally good agreement at different values of $x$ and for the $z$ component of the perturbed velocity. 

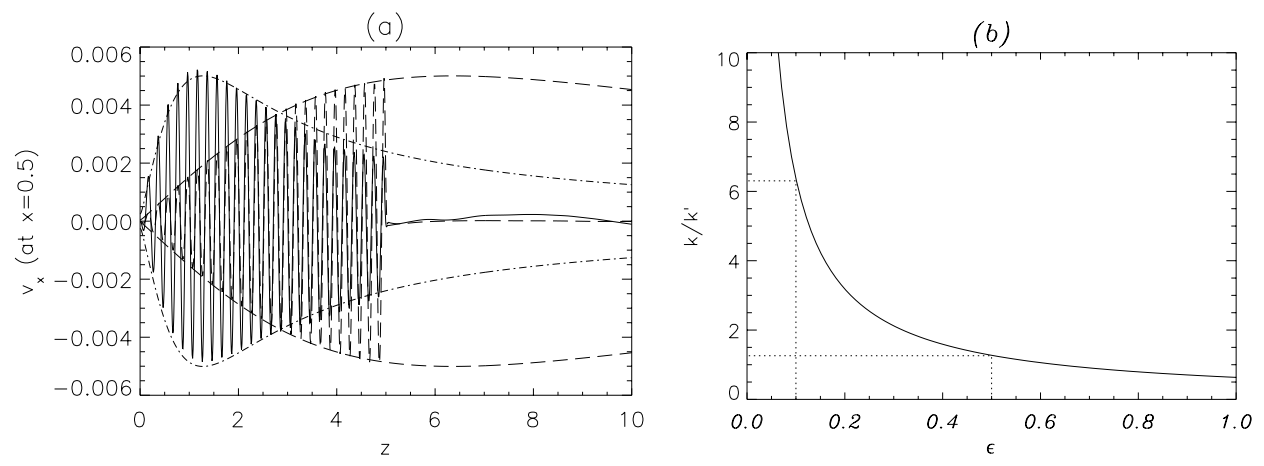

Fig. 6. a) A cross-section of the $x$ component of the perturbed velocity at $x=0.5$ and $t=50.2$, with $\varepsilon=0.5$ (solid line) and $\varepsilon=0.1$ (dashed line), $\beta=0.01$ and $\omega=\pi$. The dot-dashed and long-dashed lines are the corresponding analytical approximations. b) The behaviour of $k / k^{\prime}$ as a function of $\varepsilon$. The dotted lines indicate the location of the maximum of $A$ for $\varepsilon$ equal to 0.1 and 0.5 , respectively.

\subsection{Small $\varepsilon$ expansion}

In this section, we assume that the parameter $\varepsilon$ is small, or, in other words, that there is only a weak density inhomogeneity present. We will expand $v_{z}$ and $v_{x}$ in powers of $\varepsilon$ and try to identify the two additional modes that were described in Sect. 3.1.

The coupled equations for $v_{x}$ and $v_{z}$ are given by Eqs. (6) and (7). Setting $v=0$ and using $c_{\mathrm{s}}^{2}=\beta v_{\mathrm{A}}^{2}$ and $v_{\mathrm{A}}^{2}=1-$ $\varepsilon \cos (\pi x)$, we get

$$
\begin{aligned}
\frac{\partial^{2} v_{z}}{\partial t^{2}} & =\beta(1-\varepsilon \cos (\pi x))\left(\frac{\partial^{2} v_{x}}{\partial z \partial x}+\frac{\partial^{2} v_{z}}{\partial z^{2}}\right) \\
\frac{\partial^{2} v_{x}}{\partial t^{2}} & =(1-\varepsilon \cos (\pi x))\left((1+\beta) \frac{\partial^{2} v_{x}}{\partial x^{2}}+\beta \frac{\partial^{2} v_{z}}{\partial x \partial z}+\frac{\partial^{2} v_{x}}{\partial z^{2}}\right) .
\end{aligned}
$$

We now expand both $v_{x}$ and $v_{z}$ in powers of $\varepsilon$, setting

$$
\begin{aligned}
& v_{z}=v_{z 0}(z, t)+\varepsilon \cos (\pi x) v_{z 1}(z, t)+\ldots, \\
& v_{x}=\varepsilon \sin (\pi x) v_{x 1}(z, t)+\ldots
\end{aligned}
$$

Substituting these expressions for $v_{x}$ and $v_{z}$ into Eqs. (18) and (19) and only considering terms of order 1 , we find

$$
\frac{\partial^{2} v_{z 0}}{\partial t^{2}}=\beta \frac{\partial^{2} v_{z 0}}{\partial z^{2}}
$$

Assuming $v_{z 0}=\sin (\omega t-k z)$, we find

$$
k=\frac{\omega}{\sqrt{\beta}}
$$

which agrees with the first part of the small-beta expansion given in expressions (14) and (15). From Eqs. (18) and (19), the equations of order $\varepsilon$ are given by

$$
\begin{aligned}
& \frac{\partial^{2} v_{z 1}}{\partial t^{2}}=\beta \frac{\partial^{2} v_{z 1}}{\partial z^{2}}+\beta \pi \frac{\partial v_{x 1}}{\partial z}-\beta \frac{\partial^{2} v_{z 0}}{\partial z^{2}} \\
& \frac{\partial^{2} v_{x 1}}{\partial t^{2}}=-\pi^{2}(1+\beta) v_{x 1}-\pi \beta \frac{\partial v_{z 1}}{\partial z}+\frac{\partial^{2} v_{x 1}}{\partial z^{2}} .
\end{aligned}
$$

A particular integral solution to these equations can be found by setting

$$
\begin{aligned}
& v_{z 1_{p i}}=a \sin (\omega t-k z), \\
& v_{x 1_{p i}}=b \cos (\omega t-k z),
\end{aligned}
$$

and substituting these expressions into Eqs. (24) and (25) we find

$a=\frac{k^{2}(\beta-1)}{\pi^{2} \beta}-\frac{\beta+1}{\beta}$

and

$b=-\frac{k}{\pi}$.

A complementary function solution to Eqs. (24) and (25) can be found by neglecting the final term on the right hand side of Eq. (24) and by assuming

$v_{z 1_{c f}}=A \sin (\omega t-K z)$,

$v_{x 1_{c f}}=B \cos (\omega t-K z)$.

The equations can be combined to give a quadratic equation for $K^{2}$,

$\left(\beta K^{2}-\omega^{2}\right)\left(\omega^{2}-\pi^{2}(1+\beta)-K^{2}\right)-\beta^{2} \pi^{2} K^{2}=0$,

and hence

$$
\begin{aligned}
K^{2}= & \frac{1}{2}\left(\omega^{2}(1+1 / \beta)-\pi^{2}\right) \\
& \pm \frac{1}{2} \sqrt{\left[\pi^{2}+\omega^{2}(1+1 / \beta)\right]^{2}-\frac{4 \omega^{4}}{\beta}} .
\end{aligned}
$$

The two solutions for $K^{2}$ correspond to two different wave modes: the positive sign gives the wave number of a slow mode $\left(K_{\text {slow }}\right)$, whereas the minus sign corresponds to a fast mode $\left(K_{\text {fast }}\right)$. Finally, expressions for the constants $A$ and $B$ can be found from the boundary conditions. Thus, the solution is made up of the driven mode plus two normal modes, corresponding to fast and slow modes. It is the combination of these modes that governs the evolution of $v_{z}$ and $v_{x}$. To illustrate this in detail, we investigate the fundamental, fast and slow, normal modes in the next section.

In Fig. 7a we have plotted the variation of $K_{\text {fast }}^{2}$ (solid line) and $K_{\text {slow }}^{2}$ (dashed line), as a function of the driving frequency $\omega$, imposed at the $z=0$ boundary (with $\beta=0.1$ ). Both the fast and slow wavenumbers increase with increasing frequency, or, in other words, the larger the driving frequency, the shorter the wavelengths of the additional fast and 
(a)

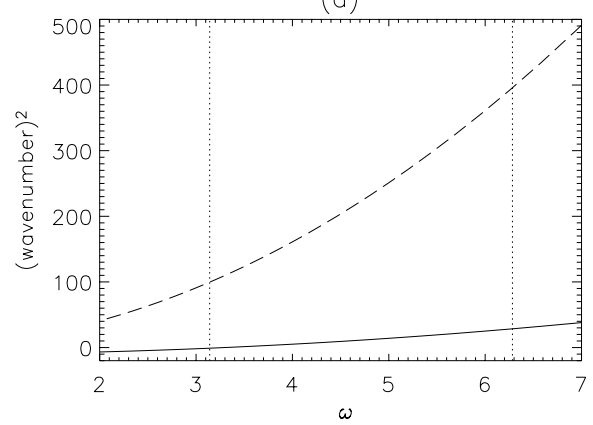

(b)

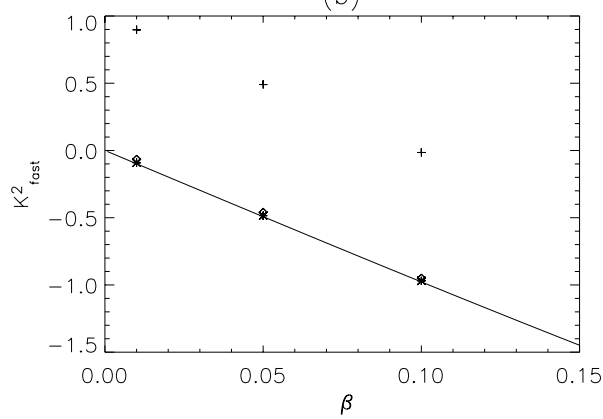

Fig. 7. a) The variation of the (squares of) the fast (solid line) and slow (dashed line) wavenumber, given by Eq. (33), as a function of the driving frequency $\omega$ and with $\beta=0.1$. The dotted lines indicate $\omega=\pi$ and $\omega=2 \pi$. b) The variation of the (square of) the fast wavenumber, given by Eq. (33), as a function of $\beta$ and with $\omega=\pi$ and with $\beta=0.1$. The symbols correspond to the values obtained for the fast wavenumber in Sect. 4.3, with $\varepsilon=0.5$ (plus), $\varepsilon=0.1$ (diamond) and $\varepsilon=0.05$ (star).

slow modes. The vertical, dotted lines indicate $\omega=\pi$ and $\omega=2 \pi$, which correspond to the numerical results described in Sect. 3.1 (Figs. 1 and 2) and Sect. 3.2 (Fig. 3), respectively. For $\omega=2 \pi$ the square of the fast and slow wavenumbers appear to be of the order of 35 and 400, which correspond to wavelengths $\sim 1.1$ (fast mode) and $\sim 0.31$ (slow mode). A comparison with the additional fast and slow modes observed in Fig. 1 (or 2) shows that these are reasonable estimates (keeping in mind that the small-epsilon expansion is probably no longer valid for $\varepsilon=0.5$ ). For $\omega=\pi$, the square of the fast wavenumber is actually negative, indicating that this mode is exponentially decaying, which explains its absence noted in Fig. 3. The slow wavelength obtained from this estimate is $\sim 0.6$, which, comparing with Fig. 3, is again of the right order. We note here that for smaller values of the inhomogeneity coefficient $\varepsilon$, there is a very good agreement between the numerical results and the fast and slow wavenumbers predicted by expression (33).

Figure $7 \mathrm{~b}$ shows the variation of the square of the fast wavenumber (solid line) with $\beta$. We see that $K_{\text {fast }}^{2}$ is negative everywhere, and hence, the fundamental fast mode would be exponentially decaying with height for all values of $\beta$. However, we do have to keep in mind that this expansion is only valid for small values of the inhomogeneity parameter $\varepsilon$.

\subsection{Normal mode interpretation}

To explain the nature of the two additional modes, we look at the normal modes of the system. For a detailed overview of such a normal mode analysis, we refer the reader to Goedbloed (1983). The ideal equations for $v_{z}$ and $v_{x}$ can be reduced to

$$
\begin{aligned}
\frac{\partial^{2} v_{z}}{\partial t^{2}} & =\beta v_{\mathrm{A}}^{2} \frac{\partial^{2} v_{x}}{\partial z \partial x}+\beta v_{\mathrm{A}}^{2} \frac{\partial^{2} v_{z}}{\partial z^{2}}, \\
\frac{\partial^{2} v_{x}}{\partial t^{2}} & =v_{\mathrm{A}}^{2}(1+\beta) \frac{\partial^{2} v_{x}}{\partial x^{2}}+\beta v_{\mathrm{A}}^{2} \frac{\partial^{2} v_{z}}{\partial x \partial z}+v_{\mathrm{A}}^{2} \frac{\partial^{2} v_{x}}{\partial z^{2}} .
\end{aligned}
$$

Assuming an $\exp (i(\omega t-K z))$ dependence for both $v_{x}$ and $v_{z}$, Eqs. (34) and (35) can be combined to give the following equation for $v_{x}$,

$$
\frac{\mathrm{d}}{\mathrm{d} x}\left[\frac{(1+\beta)\left(\omega^{2}-\beta v_{\mathrm{A}}^{2} K^{2} /(1+\beta)\right)}{\omega^{2}-\beta v_{\mathrm{A}}^{2} K^{2}} \frac{\mathrm{d} v_{x}}{\mathrm{~d} x}\right]+\left(\frac{\omega^{2}}{v_{\mathrm{A}}^{2}}-K^{2}\right) v_{x}=0 .
$$

This equation has to be solved, subject to the boundary conditions $v_{x}=0$ at $x=0$ and $x=1$. The behaviour of the solution will strongly depend on the factor in front of the first derivative term of Eq. (36). We remind the reader here that in our notation, $c_{\mathrm{s}}^{2}=\beta v_{\mathrm{A}}^{2}$ and $c_{\mathrm{T}}^{2}=c_{\mathrm{s}}^{2} v_{\mathrm{A}}^{2} /\left(c_{\mathrm{s}}^{2}+v_{\mathrm{A}}^{2}\right)=\beta v_{\mathrm{A}}^{2} /(1+\beta)$. Hence, Eq. (36) can be rewritten as

$\frac{\mathrm{d}}{\mathrm{d} x}\left[\frac{(1+\beta)\left(\omega^{2}-c_{T}^{2} K^{2}\right)}{\omega^{2}-c_{\mathrm{S}}^{2} K^{2}} \frac{\mathrm{d} v_{x}}{\mathrm{~d} x}\right]+\left(\frac{\omega^{2}}{v_{\mathrm{A}}^{2}}-K^{2}\right) v_{x}=0$.

Unlike Goedbloed (1983), we will assume $\omega$ is fixed and that the eigenvalue is $K$. From Eq. (37), it is clear that there is a slow, continuous spectrum present, with $\min \left(\omega / c_{\mathrm{T}}\right)<K<$ $\max \left(\omega / c_{\mathrm{T}}\right)$. Apart from this continuous spectrum, there are two further sets of discrete modes. The first one contains an infinite number of $K$-values, situated between $\min \left(\omega / c_{\mathrm{s}}\right)$ and $\min \left(\omega / c_{\mathrm{T}}\right)$. These correspond to slow modes, where the smallest value (i.e. the value of $K$ closest to $\min \left(\omega / c_{\mathrm{s}}\right)$ ) corresponds to the fundamental slow mode of the system and where the higher harmonics will eventually accumulate at $\min \left(\omega / c_{\mathrm{T}}\right)$. Similarly, at smaller $K\left(<\max \left(\omega / v_{\mathrm{A}}\right)\right)$, there is an infinite number of fast waves present. For the normal, fast modes, $K^{2}$ can be negative, indicating that some of the harmonics of the fast normal modes are exponentially decaying, rather than propagating. The largest of these $K_{\text {fast }}$ values will correspond to the fundamental fast mode, whereas the higher harmonics will accumulate at $-\infty$. Theoretically, the full solution would be given by summing over the infinite number of $K$ 's and including the continuum modes. In practice, we will only look for the values of $K$ corresponding to the fundamental slow and fast mode, by solving Eq. (37) numerically, subject to the boundary conditions above. To avoid confusion, we remind the reader that these are the fundamental (i.e. there are no nodes inside the domain) modes in the $x$-direction, but that the obtained wavenumber $K$ is the wavenumber in the $z$-direction.

To illustrate the above, we reconsider the numerical simulation with $\varepsilon=0.5, \beta=0.1$ and $\omega=2 \pi$ (see Fig. 2). In this case, we find $K_{\mathrm{f}}=5.98$ and $K_{\mathrm{s}}=16.40$, where the subscript "f" an "s" refer to "fast" and "slow" respectively. Figure 8a shows the $x$-dependence of the amplitude of the $x$ component of the fundamental fast (solid line) and slow (dashed line) mode. Comparing this with Fig. 1, there is a very good agreement 

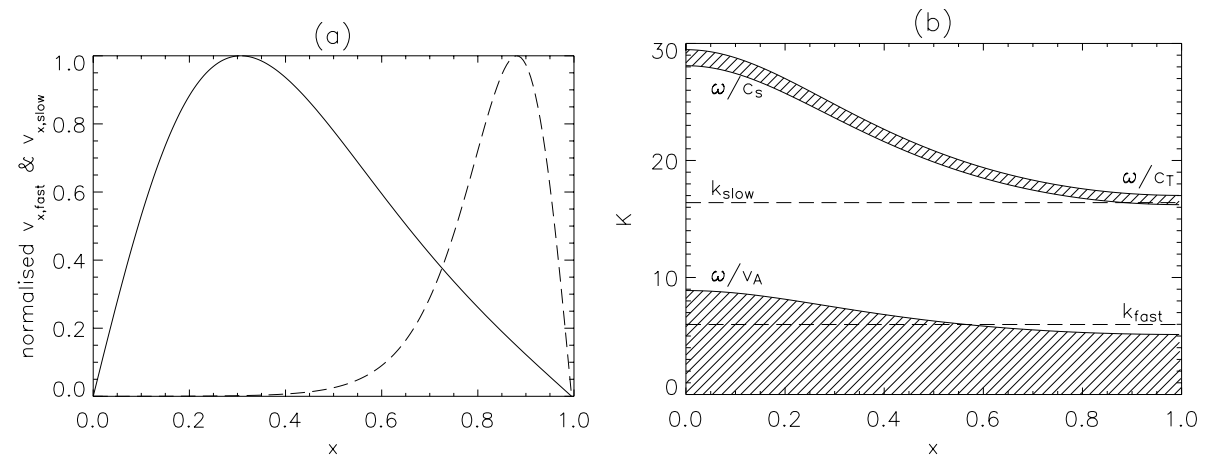

Fig. 8. a) A normalised cross-section (at constant height) of the $x$ component of the fundamental fast (solid) and slow (dashed) modes. b) Profiles of $\omega / v_{\mathrm{A}}, \omega / c_{\mathrm{S}}$ and $\omega / c_{\mathrm{T}}$ for $\varepsilon=0.5, \beta=0.1$ and $\omega=2 \pi$. The long-dashed lines correspond to the fundamental fast and slow mode wavenumbers.

(a)

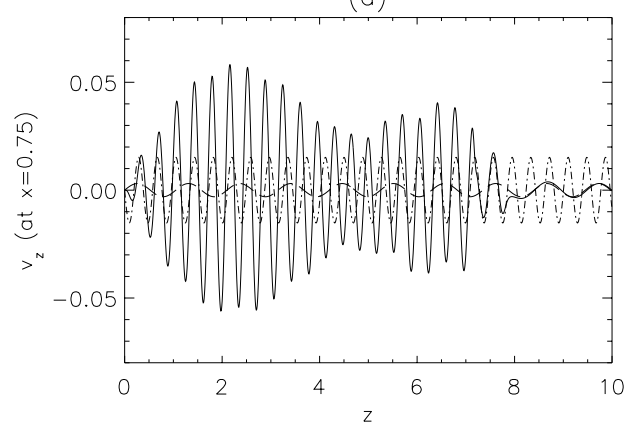

(b)

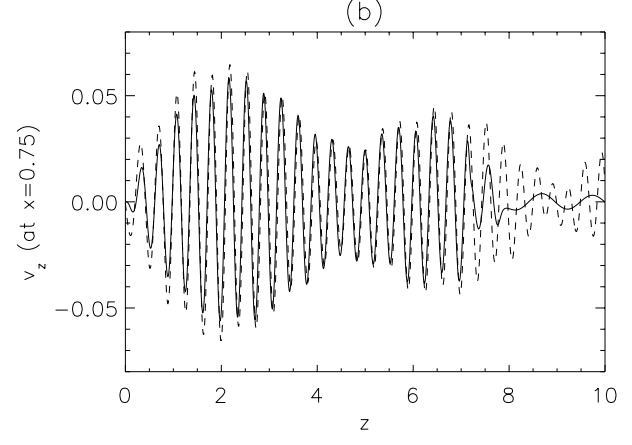

Fig. 9. a) A cross-section of the $x$ component of the perturbed velocity at $x=0.75$ and $t=20.2$, with $\varepsilon=0.5, \beta=0.1$ and $\omega=2 \pi$. The dot-dashed line corresponds to the normal slow mode, whereas the long-dashed line is the normal fast mode. b) The same cross-section of $v_{x}$. The dashed line is obtained by adding the appropriate small $\beta$ expansion and the normal modes.

between the maxima of these amplitudes, and the location at which the two extra disturbances, discussed in Sect. 3.1, occurred. The longer-wavelength, fast wave is mainly situated in the left half of the domain and the fundamental slow wave, is confined to a more narrow region near the right hand edge of the domain. Note also that the wavenumbers $K_{\mathrm{f}}$ and $K_{\mathrm{S}}$ are independent of $x$ and hence, that the fundamental fast and slow modes will propagate uniformly, despite the presence of a density inhomogeneity, as was seen in all numerical results.

Figure $8 \mathrm{~b}$ shows the profiles of $\omega / v_{\mathrm{A}}, \omega / c_{\mathrm{S}}$ and $\omega / c_{\mathrm{T}}$ for this particular example $(\varepsilon=0.5, \beta=0.1, \omega=2 \pi)$. The shaded areas indicate where the solution to Eq. (37) will be oscillatory. In the non-shaded regions, the solution will be exponentially decaying. As both $K_{\mathrm{f}}$ and $K_{\mathrm{s}}$ cut through shaded and nonshaded regions, the fundamental modes will be oscillating (in the $x$-direction) in some parts of the domain, and decaying exponentially (in the $x$-direction) in the other parts.

Figure 9a shows the same cross-section of the $x$ component of the perturbed velocity, at $x=0.75$ as Fig. $2 \mathrm{f}$. The two extra disturbances noted in Sect. 3.1 can be recognised in this figure as the longer-wavelength, small-amplitude oscillation and the few oscillations just ahead of the driven wave, which appear slightly phase-shifted. Overplotted are the fits of the fast (long-dashed) and slow (dot-dashed), fundamental modes. Both wavenumbers $K_{\mathrm{f}}$ and $K_{\mathrm{s}}$ give a remarkably good agreement. In Fig. 9b, we have combined all the analytical approximations we have found so far. The dashed line is obtained by adding the appropriate small-beta expansion and the fundamental slow and fast modes together. Although the smallbeta expansion is not strictly valid for this value of $\beta=0.1$, the combination of our approximations gives a surprisingly good agreement with the numerical result. The difference at $z>7$ between this approximate solution and the numerical result is simply caused by the fact that, at $t \approx 20$, the driven disturbance has only reached a height $z \approx 7$, whereas the approximate solution is taken as an infinite harmonic oscillation. A similarly good fit was obtained for both $v_{x}$ and $v_{z}$, at all values of $x$. Although the amplitudes do not match exactly, the approximate solution does correctly predict all the different features that occurred in the numerical results.

For a smaller driving frequency $\omega=\pi$, we obtained $K_{\mathrm{s}}=8.26$, which is the wavenumber of the harmonic oscillation that was overplotted as a dot-dashed line in Figs. 3c and d. As already discussed in the previous section, a smaller value of $\omega$ results in a smaller wavenumber. Additionally, we found that the maximum of the fundamental slow mode was situated more towards the centre of the domain, which was also noted in the corresponding numerical results (Sect. 3.2 - Fig. 3). In agreement with the results of the small-epsilon expansion, the fast wavenumber obtained in this case is negative, and hence, the fundamental fast mode decays exponentially (with height).

As a final example, we overplotted the outline of the appropriate analytical solution $(\varepsilon=0.5, \beta=0.01$ and $\omega=\pi)$ in Fig. 4, and again, it is clear that the approximate solution correctly predicts the behaviour of the numerical result. For this smaller value of $\beta$, the frequency $\omega=\pi$ results in a small (but 
positive) value for the fast wavenumber, and hence, a relatively long wavelength for the fundamental fast mode, as can be seen in Fig. 4.

We now briefly return to Fig. $7 b$, in particular to the symbols overplotted on this graphs. They represent the values of $K_{\mathrm{f}}^{2}$ that were obtained for different $\varepsilon$ and $\beta$. For $\varepsilon=0.05$ (stars) and $\varepsilon=0.1$ (diamonds), these values agree extremely well with the small-epsilon expression for $K_{\mathrm{f}}$ (Eq. (33)). For $\varepsilon=0.5$ (plus-signs), the small-epsilon expansion breaks down and the obtained values no longer agree. However, although the quantitative values no longer agree, the variation of the fast wavenumber with $\beta$ is still very similar. From Fig. 8b we see that we only obtained a positive value for $K_{\mathrm{f}}^{2}$ for a large inhomogeneity ( $\varepsilon=0.5)$ but a small plasma beta $(\beta \leq 0.1)$.

\section{Discussion and conclusions}

In this paper, we investigated the propagation of slow magnetoacoustic waves, in a 2D model of a low-beta plasma, in the presence of a horizontal density inhomogeneity. This density inhomogeneity results in a varying wave speed, causing the wave front to turn as the waves propagate with height. This "phase mixing of slow waves" is similar to phase mixing Alfvén waves, but there are some fundamental differences. As already pointed out before, unlike Alfvén waves, slow MHD waves are slightly dispersive, and not restricted to propagating along the magnetic field lines. When a density inhomogeneity is present, it is not possible to uncouple the slow and fast waves. From certain numerical simulations, one gets the impression that the driven disturbances break down into their harmonic components as they propagate up in height. However, the main aim of this paper is to complete the study of De Moortel \& Hood $(2003,2004)$ to explain the rapid damping of observed, longitudinal oscillations. A in-depth study of phase mixing of slow waves is therefore beyond the scope of this paper and will be addressed in a later paper (De Moortel et al. 2004).

The work presented here can be seen as complimetary to the study of Rosenthal et al. (2002) and Bogdan et al. (2003). These authors numerically investigated the behaviour of the different wave modes as they propagate through the solar atmosphere, resulting in a complicated picture of the various waves' characteristics and interaction. Although such numerical simulations are useful to address the complex and dynamic nature of the solar atmosphere, our simplified approach takes a step back, and concentrates solely on the propagation of slow waves in a coronal environment. This simple model allows us to isolate the effect of the various parameters. Additionally, analytical approximations to the numerical results can be obtained, enhancing our insight further.

Given the complicated nature of some of the numerical results, it is not straightforward to summarise our results. A basic feature that is always present is the turning of the wavefront, and the coupling of the driven, longitudinal component, $v_{z}$, to a horizontal component $v_{x}$. We found that the amplitude of this generated $v_{x}$ component scales with the plasma beta, which implies that the efficiency of the mode coupling is mainly governed by the size of $\beta$. Indeed, when the plasma beta is decreased, the relative importance of (plasma) pressure perturbations decreases, making it harder to generate compressional disturbances. This agrees with the results of Rosenthal et al. (2002) and Bogdan et al. (2003) who found the mode coupling to be most effective near the $\beta \approx 1$ region. In general, we found that the behaviour and interaction of both components of the perturbed velocity was very sensitive to the input parameters $(\beta, \varepsilon$ and $\omega)$. Not unexpectedly, most of the observed features were more pronounced for a larger density gradient (i.e. a larger value of the inhomogeneity coefficient $\varepsilon$ ). Increasing the driving frequency $\omega$ resulted in a larger wavelength perturbation, as did increasing the plasma beta.

One of the more surprising results of our simulations was the appearance of the fundamental fast and slow, global modes. We correctly predicted the wavenumbers of these fundamental modes, using analytical approximations and a normal mode approach. However, the exact reason of their excitation remains unclear, and the possibility that they are generated by our choice of boundary conditions cannot be excluded. In the small-epsilon expansion of Sect. 4.2, the global wavenumbers are present in the complementary function part of the solution to the coupled, partial differential equations. This suggests it might be possible to choose certain boundary conditions for which the complementary functions, and hence, the global modes would be eliminated from the solution. However, the choice of boundary conditions would be extremely specific and thus, very unlikely to have any useful, physical applications. Therefore, we did not consider this choice of boundary conditions, but retained our more general set of conditions. Under most coronal conditions, the value of the plasma beta is expected to be relatively small $(\leq 0.1)$, in which case our results suggest that the (linear) coupling of slow waves to fast waves would not be an efficient mechanism. The mode coupling is unlikely to cause a significant decay of the slow wave amplitudes, and will certainly not be sufficient to explain the rapid damping of disturbances propagating along coronal loops, observed by TRACE. These results agree with the corresponding results for Alfvén waves, presented by Botha et al. (2000). However, it is interesting to note that these authors also found a modulation of the driven disturbances, which was not addressed in their study. Looking at the $v_{x}$ component generated by one of their numerical simulations (their Fig. 9), there is a striking resemblance with the $v_{x}$ components generated in our study. It seems likely that the amplitude modulation is the result of a similar interaction between the driven disturbance and the global mode(s).

Including viscosity did result in a more rapid decay of the slow wave amplitudes. For the longitudinal component of the perturbed velocity, we found a damping rate $v_{z} \sim$ $\exp \left(-\omega v \rho_{0}\left(k^{2} z+k^{\prime 2} z^{3} / 3\right) / 2 k \beta \sqrt{\beta}\right)$. This has the same form as the damping rate for phase mixed Alfvén waves (Heyvaerts \& Priest 1983), where, at larger heights, the damping will be dominated by the $z^{3}$ component. Ofman \& Aschwanden (2002) found excellent agreement between the damping time predicted by phase mixing (of Alfvén waves) and the observed damping of transversal coronal loop oscillations, despite the fact that these observed oscillations are unlikely to be Alfvén waves. This is, however, in agreement with our results, which suggest that the damping rate of other magneto-acoustic waves due to 
phase mixing is very similar to the damping rate of phase mixed Alfvén waves.

We now return to the original question which we set out to address in this paper. Can the presence of a density inhomogeneity, which leads to mode coupling and phase mixing, explain the observed, rapid damping of slow waves in coronal loops? To answer this question, we must first of all determine whether our choice of parameters is a reasonable representation of the observed coronal conditions. An inhomogeneity parameter $\varepsilon=0.5$ would result in a density inside the coronal loop that is a factor of 3 higher than the surrounding plasma, which does not appear to be an unreasonable estimate. The coronal plasma is expected to be largely dominated by the magnetic field, and hence, a plasma beta $\beta \approx 0.1$ is an appropriate choice. The one parameter used in our simulations that has to be modified strongly to correspond with the observed oscillations is the wavelengths of the driven disturbance. We can achieve this by adapting the driving frequency $\omega$. Using a coronal sound speed $\sim 150 \mathrm{~km} \mathrm{~s}^{-1}$, the basic speed in our model is given by $v_{0}=c_{\mathrm{s}} / \sqrt{\beta} \approx 500 \mathrm{~km} \mathrm{~s}^{-1}$. Assuming an average loop radius of the order of $5 \mathrm{Mm}$ (De Moortel et al. 2002a), we obtain a basic timescale $t=L / v_{0} \approx 10 \mathrm{~s}$. To model the observed 5-min oscillations, we would require a dimensionless driving frequency $\omega \approx 2 \pi / 300 \times 10 \approx 2 \pi / 30$, which is much smaller than the driving frequencies used in our numerical simulations. However, we can estimate the expected decay of the longitudinal oscillations from some of the analytical approximations. The damping rate of the phase mixed slow waves is given by

$v_{z} \sim \exp \left(-\frac{\omega v \rho_{0}}{2 k \beta \sqrt{\beta}}\left(k^{2} z+\frac{k^{\prime 2} z^{3}}{3}\right)\right)$,

which will scale as $\mathrm{e}^{-\omega^{2}}$, seeing that $k \sim \omega$. Therefore, reducing the driving frequency, will result in a significant increase in the damping length. The observed perturbations usually decreased by a factor of about 4 , in the first 10-20 Mm along the loop. Using the same parameters as in Sect. 3.4 (but with $\omega=2 \pi / 30$ ) and the above expression for $v_{z}$, we find that, at $x=0.5, v_{z}$ would have decreased by a factor of 4 after about $100 \mathrm{Mm}$. As the phase mixing process is most efficient at the location of the largest density gradient (which in our case is at $x=0.5$ ), the amplitude decay will be even slower at the centre of the loop (i.e. near $x=0$ ). Overall, it is very unlikely that phase mixing and mode coupling, caused by the horizontal density inhomogeneity, will be able to explain the rapid, observed damping.

Although this model is unlikely to explain the damping of the observed oscillations, many interesting, and unexpected features in the numerical results deserve further study.
In particular, the relation between "phase mixing of slow MHD waves" and the generation of the global modes will be the subject of a further paper.

Acknowledgements. The authors would like to thank Dr. A. De Groof and Dr. V. Nakariakov for useful discussion and critical reading of the manuscript.

\section{References}

Berghmans, D., \& Clette, F. 1999, Sol. Phys., 186, 207

Bogdan, T. J., Carlsson, M., Hansteen, V., et al. 2003, ApJ, 599, 626

Botha, G. J. J., Arber, T. D., Nakariakov, V. M., \& Keenan, F. P. 2000 , A\&A, 363, 1186

Cally, P., \& Maddison, S. T. 1997, J. Plasma Physics, 57, 591

Cally, P. 2001, ApJ, 548, 473

DeForest, C. E., \& Gurman, J. B. 1998, ApJ, 501, L217

De Moortel, I., Ireland, J., \& Walsh, R. W. 2000, A\&A, 355, L23

De Moortel, I., Ireland, J., Hood, A. W., \& Walsh, R. W. 2002a, Sol. Phys., 209, 61

De Moortel, I., Hood, A. W., Ireland, J., \& Walsh, R. W. 2002b, Sol. Phys., 209, 89

De Moortel, I., \& Hood, A. W. 2003, A\&A, 408, 755

De Moortel, I., \& Hood, A. W. 2004, A\&A, 415, 705

De Moortel, I., et al. 2004, in preparation

Erdélyi, R. 1996, Ph.D. Thesis, K. U. Leuven

Ferraro, V. C. A., \& Plumpton, C. 1958, ApJ, 127, 459

Heyvaerts, J., \& Priest, E. R. 1983, A\&A, 117, 220

Hood, A. W., Brooks, S. J., \& Wright, A. N. 2002, Proc. R. Soc. Lond., 458, 2307

King, D. B., Nakariakov, V. M., Deluca, E. E., Golub, L., \& McClements, K. G. 2003, A\&A, 404, L1

Malara, F., Primavera, L., \& Veltri, P. 1996, ApJ, 459, 347

Nakariakov, V. M., Roberts, B., \& Murawski, K. 1997, Sol. Phys., 1753, 93

Nakariakov, V. M., Roberts, B., \& Murawski, K. 1998, A\&A, 332, 795

Nakariakov, V. M., Verwichte, E., Berghmans, D., \& Robbrecht, E. 2000, A\&A, 362, 1151

Nocera, L., Leroy, B., \& Priest, E. R. 1984, A\&A, 133, 387

Ofman, L., Romoli, M., Poletto, G., Noci, C., \& Kohl, J. L. 1997, ApJ, 491, L111

Ofman, L., Nakariakov, V. M., \& DeForest, C. E. 1999, ApJ, 514, 441

Ofman, L., Romoli, M., Poletto, G., Noci, C., \& Kohl, J. L. 2000, ApJ, 529,592

Ofman, L., \& Aschwanden, M. J. 2002, ApJ, 576, L153

Robbrecht, E., Verwichte, E., Berghmans, D., et al. 2001, A\&A, 370, 591

Rosenthal, C. S., Bogdan, T. J., Carlsson, M., et al. 2002, ApJ, 564, 508

Tsiklauri, D., \& Nakariakov, V. M. 2001, A\&A, 379, 1106

Zhugzhda, Y. D., \& Dzhalilov, N. S. 1984, A\&A, 112, 16 